\title{
L'UdPPC et la SFP publient un numéro commun sur le rayonnement synchrotron
}
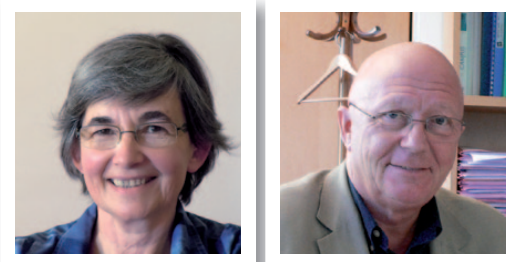

En octobre 2010, la Société française de physique et I'Union des professeurs de physique et de chimie avaient uni leurs forces éditoriales pour vous présenter un numéro hors-série à l'occasion du cinquantenaire du laser.

Ce nouveau numéro spécial ne coïncide avec aucun anniversaire particulier, mais il a semblé nécessaire à nos deux sociétés de consacrer une publication à cette autre source de lumière qui permet aux chercheurs et chercheuses de sonder la matière : les synchrotrons et leur rayonnement aux propriétés exceptionnelles.

La France héberge deux des grands synchrotrons mondiaux utilisés comme sources de rayonnement au bénéfice de toutes les disciplines. Comme les grands accélérateurs de particules, ce sont des machines au service de la communauté scientifique internationale, qui bénéficie de ces équipements pour y réaliser une recherche dans les domaines les plus variés.

La proximité de I'ESRF et de SOLEIL sur notre territoire est une opportunité unique pour donner la parole aux chercheurs qui utilisent ces machines. Ils sont les mieux placés pour nous en faire découvrir la puissance et la polyvalence, de l'analyse de la matière jusqu'aux applications biologiques et médicales. À travers leurs articles, le lecteur aura ainsi un accès privilégié à tout un pan de la science contemporaine.

Il faut souligner que la présente publication s'inscrit dans un ensemble d'initiatives communes prises par nos deux sociétés depuis de nombreuses années.

En juin 2005, à I'occasion de I'Année mondiale de la physique, la SFP et I'UdPPC avaient publié en commun un numéro hors série du Bup et du Bulletin de la Société française de physique, ancêtre des Reflets de la physique. Avec celui consacré au laser, ces deux numéros mettaient en relief quelques-uns des champs d'activité des physiciens d'aujourd'hui et leurs liens avec les disciplines les plus diverses.

Ces publications conjointes ne représentent pas la seule action commune des deux associations. Les lecteurs assidus de nos bulletins se rappellent sûrement qu'en 1986 était née une commission mixte UdPPC-SFP sur l'enseignement. Elle a poursuivi ses travaux pendant de longues années, mais fut surtout le lieu d'élaboration du projet des Olympiades de Physique France. Celui-ci vit le jour en septembre 1991, lors du

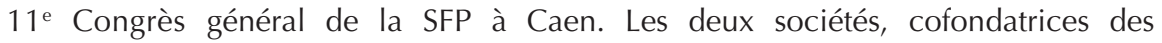
Olympiades, en sont aussi les co-organisatrices et les deux revues se font chaque année l'écho du concours. Au-delà de cet exemple, notre volonté de collaboration est constamment réaffirmée à travers des échanges réguliers, des articles publiés en commun et aussi chaque fois que sont malmenées les conditions faites à l'enseignement des sciences au lycée.

Nos remerciements s'adressent à tous les auteurs qui ont accepté de contribuer à ce beau numéro, que nous sommes heureux de vous présenter et qui devrait faire référence.

Micheline Izbicki

Présidente de l'UdPPC
Michel Lannoo

Président de la SFP 